\title{
Knowledge representation in the social Semantic Web
}

\author{
Katrin Weller \\ De Gruyter Saur \\ Berlin \\ 2010 \\ vii $+442 \mathrm{pp}$
}

ISBN 978-3-598-25180-1

£59.95

\author{
George Macgregor \\ Information Management \& Systems \\ Liverpool John Moores University
}

Keywords knowledge representation, indexing, ontology, Semantic Web

Knowledge representation in the Social Semantic Web is one of a series of scholarly monographs published as part of the 'Knowledge \& Information: studies in information science' (K\&l) book series. These are a series of peer-reviewed texts exploring some of the most exciting areas of information science, particularly areas pertaining to knowledge organisation, information retrieval, collaborative tagging and information behaviour. The last book to be published as part of the K\&l series was Folksonomies: indexing and retrieval in Web 2.0 (Peters, 2009) and, to some extent, Katrin Weller's current contribution picks up where her colleague left off.

In Knowledge representation in the Social Semantic Web Weller explores the gap between formal knowledge representation (e.g. ontologies, knowledge organisation systems, etc.) and user-generated knowledge representation techniques (i.e. folksonomies / collaborative tagging). Indeed, it remains a curious contradiction that at the same time we have witnessed a huge growth in - and deployment of - techniques to formally model data, information and knowledge structures on the Web (normally underpinned by the formats and technologies that facilitate the Semantic Web), we have also witnessed the proliferation of informal knowledge organisation schemes, normally offered via 'collaborative information services' (Peters, 2009). It is this contradiction that Weller seeks to reconcile, and her book is ultimately motivated by our need to close the gap between folksonomies and ontologies, thus ushering in an era of the so-called 'Social Semantic Web'. Of course, Weller concedes that closing this gap will be difficult and will inevitably require more research; but she also optimistically acknowledges that researchers are already equipped with many of the tools and techniques required to make it a reality.

The book is divided into three extremely detailed chapters, often exceeding 100 pages each. Chapters one and two essentially lay the foundations for chapter three by discussing knowledge representation and ontologies in the Semantic Web respectively. Chapter one ('Knowledge representation and indexing: background and future') therefore introduces the concept of knowledge representation by explaining the functions of knowledge organisation systems (KOS), indexing, metadata and their relationship to information retrieval. This chapter begins with a detailed exposition of various KOS (e.g. classifications, thesauri, facetted taxonomies, ontologies, etc.) and numerous example use cases. The second half of the chapter seeks to highlight the shared traditions of these aforementioned techniques and the Semantic Web. Key principles and technologies of the Semantic Web are explained (e.g. RDF, RDFS, URIs, SPARQL, etc.) and formal ontology languages are also briefly explored (e.g. OWL). Chapter one concludes by examining the Social Web and critically evaluating the various knowledge representation approaches which have become associated with Web 2.0. This includes observing current research efforts to improve semantics in the Social Web and the opportunities that remain to be exploited to facilitate a 'Social Semantic Web'. Chapter two ('Ontologies: semantics for 
the Web') is less diverse in its topic coverage but is nonetheless as detailed, focusing primarily on the issue of semantics in knowledge representation. This necessitates a comprehensive examination of ontologies, their peculiar characteristics and complexity, ontology languages, and opportunities for their deployment in the Social Semantic Web.

The raison d'etre of the book is encapsulated in chapter three ('Ontology engineering in the era of the Social Semantic Web'). Ontology engineering is discussed at length, as the chapter title would suggest; but Weller's mission is to better understand ontology engineering within the context of the Social Semantic Web. Consequently, chapter three provides interesting analyses of how user communities can participate in formal ontology development and how the Web 2.0 footprints left by users can be harvested and exploited to enhance knowledge representation on the Web. Interestingly, Weller draws upon her recent 'tag gardening' research conducted under the auspices of the tagCare project to explore approaches to 'ontology gardening' (e.g. techniques to weed and seed, fertilize, and harvest ontologies). Of these techniques, harvesting appears to offer most potential in the Social Semantic Web; extracting semantic relations from existing folksonomies, knowledge resources (e.g. Wikipedia), portals, and indexing and abstracting services to enhance formally created ontologies. But, as always, much work is still required, and Weller's conclusion pulls together the relevant threads to offer an exhaustive research agenda; a veritable call to arms for the information science community.

Katrin Weller is a researcher and lecturer at the Department of Information Science at Heinrich-HeineUniversity, Düsseldorf and is active in areas pertaining to the Social Semantic Web, ontology engineering, social tagging and knowledge representation. Indeed, her knowledge and extensive research experience is brought to bear in Knowledge representation in the Social Semantic Web, with each chapter citing literally hundreds of papers and incorporating a mountain of research literature into its explanations and examples. And the examples too are worth commendation; just about every concept that Weller introduces is supported by numerous examples from the 'wild', whether this be interface screen dumps from the plethora of Web 2.0 information tools that are discussed, extracts from KOS, snippets of RDF/OWL or from Protégé, model diagrams or RDF graphs. Of course, Weller's experience as a lecturer - as well as her command of the topic area generally - means that complex ideas and concepts are explained clearly, with reference to the aforementioned examples and literature. This is makes the book particularly attractive to those readers who may be less au fait with the topic area, e.g. postgraduate students; but it also includes a comprehensive index and an tremendously useful index of names, thus allowing the research orientated reader to mine the book for academic nuggets.

Like previous K\&I publications, Knowledge representation in the Social Semantic Web is well edited and beautifully typeset. It is valuable text for postgraduate students and researchers alike, and is highly recommended. The title is also available as an ebook.

\section{References}

Peters, I. (2009), Folksonomies : indexing and retrieval in Web 2.0, De Gruyter Saur, Berlin. 